\title{
ON MODIFIED BURR III-POWER DISTRIBUTION: DEVELOPMENT, PROPERTIES, CHARACTERIZATIONS, AND APPLICATIONS
}

\author{
Fiaz AHMAD BhatTI ${ }^{\star}$ \\ National College of Business Administration and Economics, Lahore, Pakistan \\ Email: fiazahmad72@gmail.com
}

\begin{abstract}
In this paper, a flexible distribution with increasing, bathtub and inverted bathtub hazard rate called Modified Burr III-Power (MBIII-Power) is developed on the basis of the generalized Pearson differential equation. The density function of MBIII-Power is arc, exponential and positively skewed shaped. Descriptive measures such as quantiles, moments, incomplete moments, inequality measures, residual life functions and reliability measures are theoretically established. The MBIII-Power distribution is characterized via different techniques. Parameters of MBIII-Power distribution are estimated using maximum likelihood method. The simulation study is performed to illustrate the performance of the maximum likelihood estimates (MLEs). Potential use of MBIII-Power distribution is demonstrated by its application to two data sets: serum-reversal time (in days) of children born from HIV-infected mothers and failure times of device data.
\end{abstract}

Keywords and phrases: Moments, Reliability, Characterizations, Maximum Likelihood Estimation

AMS Classification:: 60E05, 62E10, 62E15, 62E20, 62H12, 68U05, 68U20

\section{Introduction}

A flexible model for the analysis of lifetime data is often attractive to the researchers. Pareto, power function, exponential and Weibull distribution are of interest due to their flexibility and simplicity. In recent decades, many continuous univariate distributions have been developed but various data sets from reliability, insurance, finance, climatology, biomedical sciences and other areas do not follow these distributions. The applications of modified, extended and generalized distributions to problems in these areas are therefore warranted.

The modified, extended and generalized distributions are obtained by the introduction of some transformation or addition of one or more parameters to the base distribution. Often these newly developed distributions provide better fit to data than its sub- and competing models. Burr (1942) suggested twelve distributions known as Burr family to fit cumulative frequency functions on frequency data.The Burr-III (BIII) distribution has wide applications in modeling insurance data in finance and business and failure time data in reliability, survival and acceptance sampling plans.

\footnotetext{
* Corresponding author

(c) Institute of Statistical Research and Training (ISRT), University of Dhaka, Dhaka 1000, Bangladesh.
} 
Many modified, extended and generalized forms of BIII distribution are available in the literature such as inverse Burr (Kleiber and Kotz, 2003), BIII(Gove et al., 2008), three-parameter Burr III (Shao et al., 2008), Dagum (Benjamin et al., 2013), modified BIII (Ali et al., 2015), McDonald BIII (Gomes et al., 2015), family of size (Sinner et al., 2016), mixture of two BIII (Moisheer, 2016), gamma Burr III (Olobatuyi et al., 2017) and gamma BIII (Cordeiro et al., 2017).

The main goal of this paper is to obtain a more flexible distribution for the lifetime applications called MBIII-Power distribution. The MBIII-Power density is arc, exponential and positively skewed shaped. The MBIII-Power distribution has increasing, bathtub and inverted bathtub hazard rate function. The flexible nature of the hazard rate function of MBIII-Power distribution helps to serve as the best alternative model to other current models for modeling real data in economics, life testing, reliability, survival analysis and other areas of research. The MBIII-Power distribution provides better fits than its sub and competing models. Our interest is to study MBIII-Power distribution in terms of its mathematical properties, applications and comparison to its sub- and competing models.

This paper is sketched into the following sections. In Section 2, MBIII-Power distribution is developed on the basis of the generalized Pearson differential equation. MBIII-Power distribution is studied in terms of basic structural properties, descriptive measures based on quantiles and submodels. In Section 3, moments, incomplete moments, inequality measures, residual and reverse residual life function; and many other properties are theoretically derived. In Section 4, MBIIIPower distribution is characterized via (i) conditional expectation, (ii) ratio of truncated moments, (iii) reverse hazard rate function and (iv) elasticity function. In Section 5, the parameters of MBIIIPower are estimated using maximum likelihood method. In Section 6, a simulation study is carried out to investigate the performance of the maximum likelihood estimates (MLEs) of parameters for the MBIII-Power distribution. In Section 7, potential application of MBIII-Power distribution is demonstrated by applying it to two data sets. Goodness of fit of the probability distribution through different methods is studied. The concluding remarks are given in Section 8.

\section{Development of MBIII-Power Distribution}

A generalized Pearson differential equation (Dunning and Hanson, 1977) is of form

$$
\frac{d f}{d t}=\frac{a_{0}+a_{1} t+a_{2} t^{2}+\ldots+a_{m} t^{m}}{b_{0}+b_{1} t+b_{2} t^{2}+\cdots+b_{n} t^{n}} f(t), \quad t \in \mathrm{R}, m, n=1,2, \ldots
$$

Taking $t=\frac{x^{\kappa}}{\lambda^{\kappa}-x^{\kappa}}$, for $0<x<\lambda, m, n=1,2, \ldots$, we arrive at

$$
\frac{d}{d x}[\ln f(x)]=\frac{\kappa \lambda^{\kappa} x^{k-1}}{\left(\lambda^{\kappa}-x^{k}\right)^{2}} \frac{a_{0}+a_{1}\left[\frac{x \kappa}{\lambda^{\kappa}-x^{\kappa}}\right]+a_{2}\left[\frac{x^{\kappa}}{\lambda^{\kappa}-x^{\kappa}}\right]^{2}+\cdots+a_{m}\left[\frac{x \kappa}{\lambda^{\kappa}-x^{\kappa}}\right]^{m}}{b_{0}+b_{1}\left[\frac{x \kappa}{\lambda^{\kappa}-x^{\kappa}}\right]+b_{2}\left[\frac{x \kappa}{\lambda^{\kappa}-x^{\kappa}}\right]^{2}+\cdots+b_{n}\left[\frac{x k}{\lambda^{\kappa}-x^{\kappa}}\right]^{n}},
$$


By taking $a_{0}=0, a_{2}=\cdots=a_{m-2}=0, a_{m}=0, b_{1}=b_{2}=b_{3}=\cdots=b_{n-1}=0, m=n=-\beta$ in (2), we arrive at

$$
\frac{d}{d x}[\ln f(x)]=\frac{\kappa \lambda^{\kappa} x^{\kappa-1}}{\left(\lambda^{\kappa}-x^{\kappa}\right)^{2}} \frac{a_{1}\left[\frac{x \kappa}{\lambda^{\kappa}-x^{\kappa}}\right]^{-1}+a_{m-1}\left[\frac{x \kappa}{\lambda^{\kappa}-x^{\kappa}}\right]^{-\beta-1}}{b_{0}+b_{n}\left[\frac{x^{\kappa}}{\lambda^{\kappa}-x^{\kappa}}\right]^{-\beta}}, 0<x<\lambda,
$$

After integrating (2.1) and simplifying further, we arrive

$$
f(x)=C \frac{\kappa \lambda^{\kappa} x^{\kappa-1}}{\left(\lambda^{\kappa}-x^{\kappa}\right)^{2}}\left(\frac{x^{\kappa}}{\lambda^{\kappa}-x^{\kappa}}\right)^{-a_{1}}\left(b_{0}+b_{n}\left(\frac{x^{\kappa}}{\lambda^{\kappa}-x^{\kappa}}\right)^{-\beta}\right)^{-\frac{1}{\beta}\left(\frac{a_{m-1}}{b_{n}}-a_{1}\right)}
$$

Using $\int_{0}^{\lambda} f(x) d x=1$, the normalizing constant is

$$
C=\frac{\beta\left(\frac{b_{0}}{b_{n}}\right)^{\frac{(\beta+1)}{\beta}-\frac{a_{1}}{\beta}-1}}{B\left(\frac{a_{1}-1}{\beta}, \frac{a_{m-1}}{\beta b_{n}}-\frac{2 a_{1}}{\beta}+\frac{1}{\beta}\right)} .
$$

Taking $a_{1}=(\beta+1), a_{m-1}=(\beta+1) \gamma+(\alpha / \gamma+1) \gamma \beta, b_{0}=1, b_{n}=\gamma$ in (2.2). Therefore we arrive at

$$
f(x)=\frac{\alpha \beta \kappa \lambda^{\kappa} x^{-\beta \kappa-1}}{\left(\lambda^{\kappa}-x^{\kappa}\right)^{-\beta+1}}\left[1+\gamma\left(\frac{x^{\kappa}}{\lambda^{\kappa}-x^{\kappa}}\right)^{-\beta}\right]^{-\frac{\alpha}{\gamma}-1}, 0<x<\lambda,
$$

which is pdf of MBIII-Power distribution and the corresponding cdf is

$$
F(x)=\left[1+\gamma\left(\frac{x^{k}}{\lambda^{k}-x^{k}}\right)^{-\beta}\right]^{-\frac{\alpha}{\gamma}}, 0 \leq x \leq \lambda,
$$

where $\alpha, \beta, \gamma, \kappa$, and $\lambda$ are positive parameters.

\subsection{Transformations and Compounding}

The MBIII-Power distribution is derived through (i) ratio of exponential and gamma random variables and (ii) compounding generalized inverse Weibull-Power (GIW-P) and gamma distributions.

Lemma 2.1. (i) If $Z_{1} \sim \exp (1)$ and $Z_{2} \sim \operatorname{gamma}(\alpha / \gamma, 1)$, then for $Z_{1}=\gamma\left[\frac{X^{k}}{\lambda^{k}-X^{k}}\right]^{-\beta} Z_{2}$, we have

$$
X=\lambda\left[1+\left(\frac{\gamma Z_{2}}{Z_{1}}\right)^{-\frac{1}{\beta}}\right]^{-\frac{1}{\kappa}} \sim \text { MBIII-Power }(\alpha, \beta, \gamma, \lambda) .
$$

(ii) If $X \sim \operatorname{GIW}-P(\beta, k, \gamma, \lambda, \theta)$ and $\theta \sim \operatorname{gamma}(\alpha / \gamma, 1)$, integrating the effect of $\theta$ with the help of

$$
f(x ; \alpha, \beta, \gamma, \lambda)=\int_{0}^{\infty} \operatorname{GIW}-P(x / \beta, \gamma, \lambda, \theta) g(\theta / \alpha, \gamma) d \theta
$$

then $X$ has MBIII-Power $(\alpha, \beta, \gamma, \lambda)$. 


\subsection{Structural Properties of MBIII-Power Distribution}

The survival, hazard, cumulative hazard and reverse hazard functions and the Mills ratio of a random variable $X$ with MBIII-Power distribution are given below for $0 \leq x \leq \lambda$

$$
\begin{aligned}
& S(x)=1-\left[1+\gamma\left(\frac{x^{\kappa}}{\lambda^{\kappa}-x^{\kappa}}\right)^{-\beta}\right]^{-\frac{\alpha}{\gamma}} \\
& h(x)=\frac{\alpha \beta \kappa \frac{\lambda^{\kappa} x^{-\beta x-1}}{\left(\lambda^{\kappa}-x^{\kappa}\right)^{-\beta+1}}\left[1+\gamma\left(\frac{x^{\kappa}}{\lambda^{\kappa}-x^{\kappa}}\right)^{-\beta}\right]^{-\frac{\alpha}{\gamma}-1}}{1-\left[1+\gamma\left(\frac{x^{\kappa}}{\lambda^{\kappa}-x^{\kappa}}\right)^{-\beta}\right]^{-\frac{\alpha}{\gamma}}} \\
& \left.r(x)=\frac{d}{d x} \ln \left\{1+\gamma\left(\frac{x^{\kappa}}{\lambda^{\kappa}-x^{\kappa}}\right)^{-\beta}\right]^{-\frac{\alpha}{\gamma}}\right\} \\
& H(x)=-\ln \left\{1-\left[1+\gamma\left(\frac{x^{\kappa}}{\lambda^{\kappa}-x^{\kappa}}\right)^{-\beta}\right]^{-\frac{\alpha}{\gamma}}\right\} \\
& m(x)=\frac{1-\left[1+\gamma\left(\frac{x^{\kappa}}{\lambda^{\kappa}-x^{\kappa}}\right)^{-\beta}\right]^{-\frac{\alpha}{\gamma}}}{\alpha \beta \kappa \frac{\lambda^{\kappa} x^{-\beta \kappa-1}}{\left(\lambda^{\kappa}-x^{\kappa}\right)^{-\beta+1}}\left[1+\gamma\left(\frac{x^{\kappa}}{\lambda^{\kappa}-x^{\kappa}}\right)^{-\beta}\right]^{-\frac{\alpha}{\gamma}-1}} .
\end{aligned}
$$

The elasticity $e(x)=\frac{d \ln F(x)}{d \ln x}$ for MBIII-Power distribution is

$$
e(x)=\frac{d}{d \ln x} \ln \left\{\left[1+\gamma\left(\frac{x^{\kappa}}{\lambda^{\kappa}-x^{\kappa}}\right)^{-\beta}\right]^{-\frac{\alpha}{\gamma}}\right\}, \quad 0<x<\lambda .
$$

The elasticity of MBIII-Power distribution shows the behavior of the accumulation of probability in the domain of the random variable.

\subsubsection{Descriptive Measures based on Quantiles}

The quantile function of MBIII-Power distribution is $x_{q}=\lambda\left[\left(\frac{q^{-\frac{\gamma}{\alpha}}-1}{\gamma}\right)^{\frac{1}{\beta}}+1\right]^{-\frac{1}{\kappa}}$.The median of MBIII-Power distribution is $x_{\text {med }}=\lambda\left[\left(\frac{2 \frac{\gamma}{\alpha}-1}{\gamma}\right)^{\frac{1}{\beta}}+1\right]^{-\frac{1}{\kappa}}$ and random number generator for MBIII-Power distribution is $X=\lambda\left[\left(\frac{Z^{-\frac{\gamma}{\alpha}}-1}{\gamma}\right)^{\frac{1}{\beta}}+1\right]^{-\frac{1}{\kappa}}$, where the random variable $Z$ has uniform distribution with domain $(0,1)$.

\subsubsection{Sub-Models}

The MBIII-Power distribution has the following sub models. 
Table 1: Sub-Models of MBIII-Power Distribution

\begin{tabular}{ccccccl}
\hline serial no. & $\alpha$ & $\beta$ & $\gamma$ & $\kappa$ & $\lambda$ & Distribution \\
\hline 1 & $\alpha$ & $\beta$ & $\gamma$ & $\kappa$ & $\lambda$ & MBIII-Power Distribution \\
2 & $\alpha$ & $\beta$ & 1 & $\kappa$ & $\lambda$ & BIII-Power Distribution \\
3 & $\alpha$ & 1 & 1 & $\kappa$ & $\lambda$ & Inverse Lomax Power (IL-Power)distribution \\
4 & 1 & $\beta$ & 1 & $\kappa$ & $\lambda$ & Log-logistic Power(LL-Power)distribution \\
5 & $\alpha$ & $\beta$ & $\gamma \rightarrow 0$ & $\kappa$ & $\lambda$ & Generalized inverse Weibull Power(GIW)distribution \\
\hline
\end{tabular}

\subsubsection{Shapes of the MBIII-Power Density and Hazard Rate Functions}

The following graphs show that shapes of MBIII-Power density are arc, exponential and positively skewed (Figure 1). The MBIII-Power distribution has increasing, inverted bathtub and bathtub hazard rate function (Figure 2).

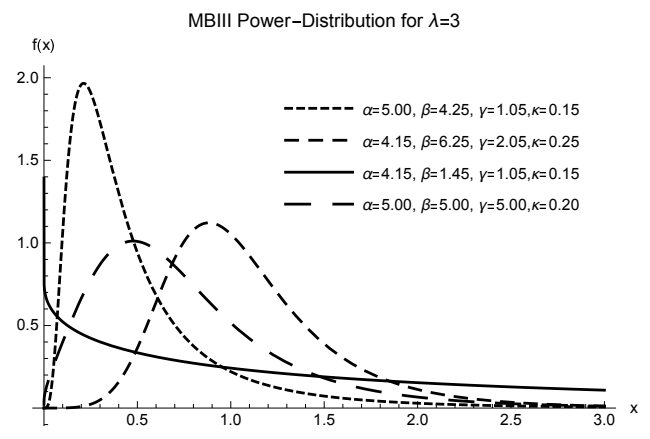

Figure 1: Plots of pdf of MBIII-Power distribution

\section{Moments}

Moments, central moments, incomplete moments, inequality measures, residual life functions and some other properties are achieved in this section.

\subsection{Moments of MBIII-Power Distribution}

The $r^{t h}$ moments about the origin of $X$ for MBIII-Power distribution are

$$
\mu_{r}^{\prime}=E\left(X^{r}\right)=\int_{0}^{\lambda} x^{r} f(x) d x=\int_{0}^{\lambda} x^{r} \frac{\alpha \beta \kappa \lambda^{\kappa} x^{-\beta \kappa-1}}{\left(\lambda^{\kappa}-x^{\kappa}\right)^{-\beta+1}}\left[1+\gamma\left(\frac{x^{\kappa}}{\lambda^{\kappa}-x^{\kappa}}\right)^{-\beta}\right]^{-\frac{\alpha}{\gamma}-1} d x
$$



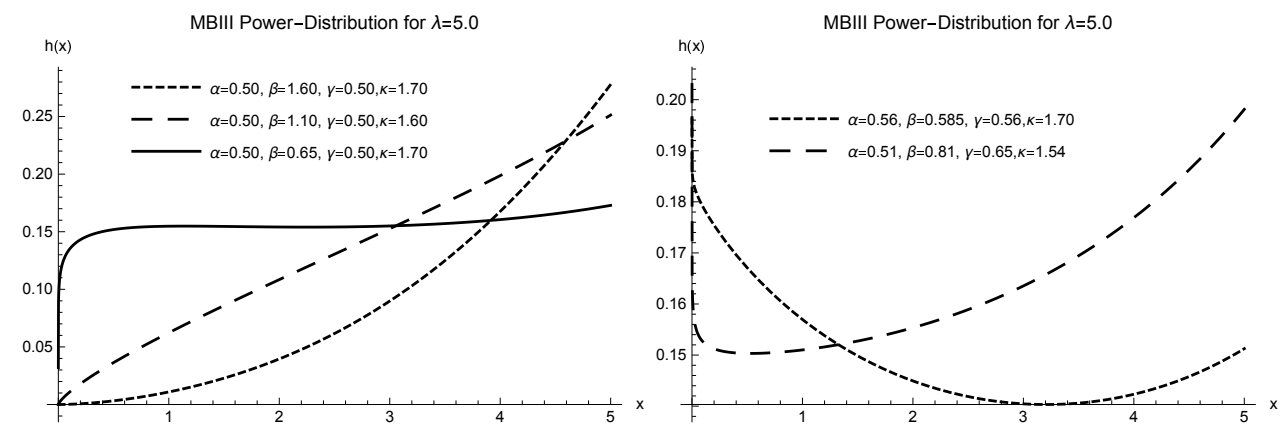

Figure 2: Plots of hrf of MBIII-Power distribution.

Let $\gamma\left(\frac{x \kappa}{\lambda^{\kappa}-x^{\kappa}}\right)^{-\beta}=w$, then we obtain

$$
x^{r}=\lambda^{r}\left[1+\left(\frac{w}{\gamma}\right)^{\frac{1}{\beta}}\right]^{-\frac{r}{\kappa}}=\lambda^{r} \sum_{\ell=0}^{\infty} \frac{(-1)^{\ell}}{\ell !}\left(\frac{r}{\kappa}\right)_{\ell} \gamma^{-\frac{\ell}{\beta}} w^{\frac{\ell}{\beta}} .
$$

For $r=1,2,3, \ldots$ we reach at

$$
\begin{aligned}
\mu_{r}^{\prime}=E\left(X^{r}\right) & =\frac{\alpha}{\gamma} \int_{0}^{\infty} \lambda^{r} \sum_{\ell=0}^{\infty} \frac{(-1)^{\ell}}{\ell !}\left(\frac{r}{\kappa}\right)_{\ell} \gamma^{-\frac{\ell}{\beta}} w^{\frac{\ell}{\beta}}[1+w]^{-\frac{\alpha}{\gamma}-1} d w \\
& =\frac{\alpha}{\gamma} \lambda^{r} \sum_{\ell=0}^{\infty} \frac{(-1)^{\ell}}{\ell !}\left(\frac{r}{\kappa}\right)_{\ell} \gamma^{-\frac{\ell}{\beta}} \int_{0}^{\infty} w^{\frac{\ell}{\beta}}[1+w]^{-\frac{\alpha}{\gamma}-1} d w \\
& =\alpha \lambda^{r} \sum_{\ell=0}^{\infty} \frac{(-1)^{\ell}}{\ell !}\left(\frac{r}{\kappa}\right)_{\ell} \gamma^{-\left(\frac{\ell}{\beta}+1\right)} B\left(1+\frac{\ell}{\beta}, \frac{\alpha}{\gamma}-\frac{\ell}{\beta}\right) .
\end{aligned}
$$

The factorial moments for MBIII-Power distribution are

$$
\begin{aligned}
E[X]_{m} & =\sum_{r=1}^{m} \varphi_{r} E\left(X^{r}\right) \\
& =\alpha \lambda^{r} \sum_{r=1}^{m} \sum_{\ell=0}^{\infty} \varphi_{r} \frac{(-1)^{\ell}}{\ell !}\left(\frac{r}{k}\right)_{\ell} \gamma^{-\left(\frac{\ell}{\beta}+1\right)} B\left(1+\frac{\ell}{\beta}, \frac{\alpha}{\gamma}-\frac{\ell}{\beta}\right),
\end{aligned}
$$

where $\left(\frac{r}{\kappa}\right)_{\ell}=\frac{\Gamma\left(\frac{r}{\kappa}+\ell\right)}{\Gamma(\ell)}$ is Pochhammar symbol, $[X]_{i}=X(X+1)(X+2) \cdots(X+i-1)$ and $\varphi_{r}$ is the Stirling number of the first kind.

The Mellin transform (Bertand et al., 1994) helps to determine moments for a probability distribution. The Mellin transform of $\mathrm{X}$ with MBIII-Power distribution is

$$
\begin{aligned}
\mathrm{M}\{f(x) ; s\}=f^{*}(s) & =\int_{0}^{\lambda} f(x) x^{s-1} d x \\
& =\int_{0}^{\lambda} x^{s-1} \frac{\alpha \beta \kappa \lambda^{\kappa} x^{-\beta \kappa-1}}{\left(\lambda^{\kappa}-x^{\kappa}\right)^{-\beta+1}}\left[1+\gamma\left(\frac{x^{\kappa}}{\lambda^{\kappa}-x^{\kappa}}\right)^{-\beta}\right]^{-\frac{\alpha}{\gamma}-1} d x .
\end{aligned}
$$


After some transformation and simplification, we reach at

$$
M\{f(x) ; s\}=\alpha \lambda^{s-1} \sum_{\ell=0}^{\infty} \frac{(-1)^{\ell}}{\ell !}\left(\frac{s-1}{\kappa}\right)_{\ell} \gamma^{-\left(\frac{\ell}{\beta}+1\right)} B\left(1+\frac{\ell}{\beta}, \frac{\alpha}{\gamma}-\frac{\ell}{\beta}\right) .
$$

The $k^{\text {th }}$ moment about mean of $X$ is determined from the relationship

$$
\mu_{k}=E[X-E(X)]^{\kappa}=\sum_{j=1}^{\kappa}\left(\begin{array}{c}
\kappa \\
j
\end{array}\right)(-1)^{j} \mu_{j}^{\prime} \mu_{(\kappa-j)}^{\prime} .
$$

The Pearson's measure of skewness $\gamma_{1}$, Kurtosis $\beta_{2}$, moment generating function and cumulants can be calculated from

$\gamma_{1}=\frac{\mu_{3}}{\left(\mu_{2}\right)^{\frac{3}{2}}}, \beta_{2}=\frac{\mu_{4}}{\left(\mu_{2}\right)^{2}}, M_{X}(t)=\sum_{r=1}^{\infty} \frac{t^{r}}{r !} E(X)^{r}$ and $k_{r}=E\left(X^{r}\right)-\sum_{c=1}^{r-1}\left(\begin{array}{c}r-1 \\ c-1\end{array}\right) k_{c} E\left(X^{r-c}\right)$.

Table 2 shows the numerical measure of the median, mean, standard deviation, skewness and Kurtosis of the MBIII-Power distribution for selected parameter values to describe their effect on these measures.

Table 2: Median, mean, standard deviation (SD), skewness and Kurtosis of the MBIII-Power distribution.

\begin{tabular}{lccccc}
\hline $\begin{array}{l}\text { Parameters } \\
(\alpha, \beta, \gamma, \kappa, \lambda)\end{array}$ & Median & Mean & SD & Skewness & Kurtosis \\
\hline $0.5,0.5,0.5,0.5,0.5$ & 0.410098 & 2.57075 & 3.47575 & 1.07902 & 2.57506 \\
$1,0.5,0.5,0.5,0.5$ & 3.51207 & 4.30882 & 3.79406 & 0.257573 & 1.44476 \\
$1.5,0.5,0.5,0.5,0.5$ & 6.19303 & 5.54611 & 3.66393 & -0.231816 & 1.48783 \\
$3,0.5,0.5,0.5,0.5$ & 8.89899 & 7.62625 & 2.78509 & -1.20246 & 3.27421 \\
$0.5,1.5,0.5,0.5,0.5$ & 1.49344 & 2.18039 & 2.10016 & 1.2648 & 4.03243 \\
$0.5,3,0.5,0.5,0.5$ & 1.9572 & 2.17245 & 1.27245 & 1.07699 & 4.59801 \\
$0.5,0.5,1.5,0.5,0.5$ & 0.0288227 & 2.1258 & 3.40947 & 1.35313 & 3.1956 \\
$0.5,0.5,3,0.5,0.5$ & 0.00252355 & 1.99428 & 3.42203 & 1.44987 & 3.4222 \\
$0.5,0.5,5,0.5,0.5$ & 0.000889997 & 2.0456 & 3.51334 & 1.41357 & 3.27633 \\
$0.5,0.5,0.5,1.5,0.5$ & 3.41794 & 4.29138 & 3.54191 & 0.345652 & 1.59308 \\
$0.5,0.5,0.5,3,0.5$ & 5.83773 & 5.76794 & 3.10004 & -0.124385 & 1.56332 \\
$0.5,0.5,0.5,5,0.5$ & 7.24012 & 6.86807 & 2.54519 & -0.454475 & 2.05579 \\
$1.5,1.5,1.5,1.5,0.5$ & 6.84914 & 6.58251 & 1.96912 & -0.570981 & 2.75425 \\
$3,1.5,1.5,1.5,0.5$ & 7.89908 & 7.69299 & 1.3877 & -0.39277 & 3.39277 \\
$3,3,3,3,0.5$ & 8.38851 & 8.30257 & 0.685927 & -0.957927 & 4.96754 \\
$5,5,2.5,2.75,0.5$ & 8.24853 & 8.25292 & 0.355688 & 0.00905782 & 3.4087 \\
$1,5,5,5,0.5$ & 8.36403 & 8.03516 & 1.08078 & -1.67318 & 6.33926 \\
\hline
\end{tabular}




\subsection{Moments of Order Statistics}

Moments of order statistics are also designed to predict the extreme events for future on the basis of few early extreme events. The pdf of $m^{t h}$ order statistic $X_{m: n}$ for MBIII-Power distribution is

$$
f_{X_{m: n}}(x)=\frac{\sum_{j=0}^{n-m}(-1)^{j}\left(\begin{array}{l}
n-m \\
j
\end{array}\right)}{B(m, n-m+1)} \frac{\alpha \beta k \lambda^{k} x^{-\beta k-1}}{\left(\lambda^{k}-x^{k}\right)^{-\beta+1}}\left[1+\gamma\left(\frac{x^{k}}{\lambda^{k}-x^{k}}\right)^{-\beta}\right]^{-\frac{\alpha(m+j)}{\gamma}-1}, x>0
$$

The $r^{t h}(r=1,2, \ldots)$ moments about the origin of $m^{t h}$ order statistic $X_{m: n}$ for MBIII-Power distribution are given by

$$
\begin{aligned}
E\left(X_{m: n}^{r}\right) & =\int_{0}^{\lambda} x^{r} \frac{\sum_{j=0}^{n-m}(-1)^{j}\left(\begin{array}{c}
n-m \\
j
\end{array}\right)}{B(m, n-m+1)} \frac{\alpha \beta k \lambda^{k} x^{-\beta k-1}}{\left(\lambda^{k}-x^{k}\right)^{-\beta+1}}\left[1+\gamma\left(\frac{x^{k}}{\lambda^{k}-x^{k}}\right)^{-\beta}\right]^{-\frac{\alpha(m+j)}{\gamma}-1} d x \\
& =\frac{\sum_{j=0}^{n-m}(-1)^{j}\left(\begin{array}{c}
n-m \\
j
\end{array}\right)}{B(m, n-m+1)} \frac{\alpha}{\gamma} \lambda^{r} \sum_{\ell=0}^{\infty} \frac{(-1)^{\ell}}{\ell !}\left(\frac{r}{k}\right)_{\ell} \gamma^{-\frac{\ell}{\beta}} B\left(1+\frac{\ell}{\beta}, \frac{\alpha}{\gamma}(m+j)-\frac{\ell}{\beta}\right)
\end{aligned}
$$

\subsection{Incomplete Moments}

Incomplete moments are used to study mean inactivity life, mean residual life function and other inequality measures. The lower incomplete moments for random variable $\mathrm{X}$ having MBIII-Power distribution are

$$
E_{X \leq z}\left(X^{r}\right)=\int_{0}^{z} x^{r} \frac{\alpha \beta \kappa \lambda^{\kappa} x^{-\beta \kappa-1}}{\left(\lambda^{\kappa}-x^{\kappa}\right)^{-\beta+1}}\left[1+\gamma\left(\frac{x^{k}}{\lambda^{k}-x^{k}}\right)^{-\beta}\right]^{-\frac{\alpha}{\gamma}-1} d x
$$

Let

$$
\gamma\left(\frac{x^{k}}{\lambda^{k}-x^{k}}\right)^{-\beta}=w \text { and } x^{r}=\lambda^{r}\left[1+\left(\frac{w}{\gamma}\right)^{\frac{1}{\beta}}\right]^{-\frac{r}{k}}=\lambda^{r} \sum_{\ell=0}^{\infty} \frac{(-1)^{\ell}}{\ell !}\left(\frac{r}{k}\right)_{\ell} \gamma^{-\frac{\ell}{\beta}} w^{\frac{\ell}{\beta}}
$$

We obtain

$$
\begin{aligned}
E_{X \leq z}\left(X^{r}\right) & =\frac{\alpha}{\gamma} \int_{\gamma\left(\frac{z^{k}}{\lambda^{k}-z^{k}}\right)^{-\beta}}^{\infty} \lambda^{r} \sum_{\ell=0}^{\infty} \frac{(-1)^{\ell}}{\ell !}\left(\frac{r}{k}\right)_{\ell} \gamma^{-\frac{\ell}{\beta}} w^{\frac{\ell}{\beta}}[1+w]^{-\frac{\alpha}{\gamma}-1} d w \\
& =\frac{\alpha}{\gamma} \lambda^{r} \sum_{\ell=0}^{\infty} \frac{(-1)^{\ell}}{\ell !}\left(\frac{r}{k}\right)_{\ell} \gamma^{-\frac{\ell}{\beta}} \int_{\gamma\left(\frac{z^{k}}{\lambda^{k}-z^{k}}\right)^{-\beta}}^{\infty} w^{\frac{\ell}{\beta}}[1+w]^{-\frac{\alpha}{\gamma}-1} d w \\
& =\alpha \lambda^{r} \sum_{\ell=0}^{\infty} \frac{(-1)^{\ell}}{\ell !}\left(\frac{r}{k}\right)_{\ell} \gamma^{-\left(\frac{\ell}{\beta}+1\right)}\left[B\left(1+\frac{\ell}{\beta}, \frac{\alpha}{\gamma}-\frac{\ell}{\beta}\right)-B_{w(z)}\left(1+\frac{\ell}{\beta}, \frac{\alpha}{\gamma}-\frac{\ell}{\beta}\right)\right]
\end{aligned}
$$

where $B_{z}(.,$.$) is incomplete beta function and w(z)=\gamma\left(\frac{x^{k}}{\lambda^{k}-x^{k}}\right)^{-\beta}$. 
The upper incomplete moments for the random variable $\mathrm{X}$ with MBIII-Power distribution are

$$
\begin{aligned}
E_{X \geq z}\left(X^{r}\right) & =\int_{z}^{\lambda} x^{r} \frac{\alpha \beta k \lambda^{k} x^{-\beta k-1}}{\left(\lambda^{k}-x^{k}\right)^{-\beta+1}}\left[1+\gamma\left(\frac{x^{k}}{\lambda^{k}-x^{k}}\right)^{-\beta}\right]^{-\frac{\alpha}{\gamma}-1} d x \\
& =\alpha \lambda^{r} \sum_{\ell=0}^{\infty} \frac{(-1)^{\ell}}{\ell !}\left(\frac{r}{k}\right)_{\ell} \gamma^{-\left(\frac{\ell}{\beta}+1\right)} B_{w(z)}\left(1+\frac{\ell}{\beta}, \frac{\alpha}{\gamma}-\frac{\ell}{\beta}\right) .
\end{aligned}
$$

The mean deviation about mean is $M D_{\bar{X}}=E\left|X-\mu_{1}^{1}\right|=2 \mu_{1}^{1} F\left(\mu_{1}^{1}\right)-2 \mu_{1}^{1} M_{1}^{\prime}\left(\mu_{1}^{1}\right)$ and mean deviation about median is $M D_{M}=E|X-M|=2 M F(M)-2 M M_{1}^{\prime}(M)$, where $\mu_{1}^{\prime}=E(X)$ and $M=Q(0.5)$. Bonferroni and Lorenz curves for a specified probability $\mathrm{p}$ are computed by $B(p)=M_{1}^{\prime}(q) / p \mu_{1}^{1}$ and $L(p)=M_{1}^{\prime}(q) / \mu_{1}^{1}$, where $q=Q(p)$.

\subsection{Residual Life Functions}

The $n t h$ moment of residual life, say $m_{n}(z)$, of $X$ with MBIII-Power distribution is

$$
\begin{aligned}
m_{n}(z) & =E\left[(X-z)^{n} \mid X>z\right] \\
& =\frac{1}{S(z)} \int_{z}^{\infty}(x-z)^{s} f(x) d x \\
& =\frac{1}{S(z)} \sum_{s=0}^{n}\left(\begin{array}{l}
n \\
s
\end{array}\right)(-z)^{n-s} E_{X>z}\left(X^{s}\right) \\
& =\frac{1}{S(z)} \sum_{s=0}^{n}\left(\begin{array}{l}
n \\
s
\end{array}\right)(-z)^{n-s} \alpha \lambda^{s} \sum_{\ell=0}^{\infty} \frac{(-1)^{\ell}}{\ell !}\left(\frac{s}{k}\right)_{\ell} \gamma^{-\left(\frac{\ell}{\beta}+1\right)} B_{w(z)}\left(1+\frac{\ell}{\beta}, \frac{\alpha}{\gamma}-\frac{\ell}{\beta}\right) .
\end{aligned}
$$

The average remaining lifetime of a component at time $z$, say $m_{1}(z)$, or life expectancy known as mean residual life (MRL) function is

$$
m_{1}(z)=\frac{1}{S(z)} \sum_{s=0}^{1}\left(\begin{array}{l}
1 \\
s
\end{array}\right)(-z)^{1-s} \alpha \lambda^{s} \sum_{\ell=0}^{\infty} \frac{(-1)^{\ell}}{\ell !}\left(\frac{s}{k}\right)_{\ell} \gamma^{-\left(\frac{\ell}{\beta}+1\right)} B_{w(z)}\left(1+\frac{\ell}{\beta}, \frac{\alpha}{\gamma}-\frac{\ell}{\beta}\right) .
$$

The $n t h$ moment of reverse residual life, say $M_{n}(z)$, of $X$ with MBIII-Power distribution is

$$
\begin{aligned}
M_{n}(z)= & E\left[(z-X)^{n} / X \leq z\right]=\frac{1}{F(z)} \int_{0}^{z}(z-x)^{n} f(x) d x \\
= & \frac{1}{F(z)} \sum_{s=0}^{n}(-1)^{s}\left(\begin{array}{c}
n \\
s
\end{array}\right) z^{n-s} E_{X \leq z}\left(X^{s}\right) \\
= & \frac{1}{F(z)} \sum_{s=0}^{n}(-1)^{s}\left(\begin{array}{c}
n \\
s
\end{array}\right) z^{n-s} \alpha \lambda^{s} \sum_{\ell=0}^{\infty} \frac{(-1)^{\ell}}{\ell !}\left(\frac{s}{k}\right)_{\ell} \gamma^{-\left(\frac{\ell}{\beta}+1\right)} \\
& \times\left[B\left(1+\frac{\ell}{\beta}, \frac{\alpha}{\gamma}-\frac{\ell}{\beta}\right)-B_{w(z)}\left(1+\frac{\ell}{\beta}, \frac{\alpha}{\gamma}-\frac{\ell}{\beta}\right)\right] .
\end{aligned}
$$


The waiting time $z$ for failure of a component has passed with condition that this failure had happened in the interval $[0, z]$ is called mean waiting time (MWT) or mean inactivity time. The waiting time $z$ for failure of a component of $X$ having MBIII-Power distribution is defined by

$$
\begin{aligned}
M_{1}(z)= & \frac{1}{F(z)} \sum_{s=0}^{1}(-1)^{s}\left(\begin{array}{l}
1 \\
s
\end{array}\right) z^{1-s} \alpha \lambda^{s} \sum_{\ell=0}^{\infty} \frac{(-1)^{\ell}}{\ell !}\left(\frac{s}{k}\right)_{\ell} \gamma^{-\left(\frac{\ell}{\beta}+1\right)} \\
& \times\left[B\left(1+\frac{\ell}{\beta}, \frac{\alpha}{\gamma}-\frac{\ell}{\beta}\right)-B_{w(z)}\left(1+\frac{\ell}{\beta}, \frac{\alpha}{\gamma}-\frac{\ell}{\beta}\right)\right] .
\end{aligned}
$$

In the following sections, reliability and uncertainty measures are studied.

\subsection{Stress-strength Reliability for MBIII-Power Distribution}

Let $X_{1} \sim \operatorname{MBIII-Power}\left(\alpha_{1}, \beta, \gamma, \kappa, \lambda\right), X_{2} \sim \operatorname{MBIII-Power}\left(\alpha_{2}, \beta, \gamma, \kappa, \lambda\right)$ such that $X_{1}$ represents strength and $X_{2}$ represents stress, then $R=\operatorname{Pr}\left(X_{2}<X_{1}\right)=\int_{0}^{\lambda} f_{x_{1}}(x) F_{x_{2}}(x) d x$ is the characteristic of the distribution of $X_{1}$ and $X_{2}$, then reliability of the component for MBIII-Power distribution is computed as

$$
\begin{aligned}
R & =\int_{0}^{\lambda} \frac{\alpha_{1} \beta k \lambda^{k} x^{-\beta k-1}}{\left(\lambda^{k}-x^{k}\right)^{-\beta+1}}\left[1+\gamma\left(\frac{x^{k}}{\lambda^{k}-x^{k}}\right)^{-\beta}\right]^{-\frac{\alpha_{1}}{\gamma}-1}\left[1+\gamma\left(\frac{x^{k}}{\lambda^{k}-x^{k}}\right)^{-\beta}\right]^{-\frac{\alpha_{2}}{\gamma}} d x \\
& =\int_{0}^{\lambda} \frac{\alpha_{1} \beta k \lambda^{k} x^{-\beta k-1}}{\left(\lambda^{k}-x^{k}\right)^{-\beta+1}}\left[1+\gamma\left(\frac{x^{k}}{\lambda^{k}-x^{k}}\right)^{-\beta}\right]^{-\frac{\alpha_{1}}{\gamma}-\frac{\alpha_{2}}{\gamma}-1} d x \\
& =\frac{\alpha_{1}}{\left(\alpha_{1}+\alpha_{2}\right)} .
\end{aligned}
$$

Therefore (i) $R$ is independent of $\beta, \gamma, \kappa$, and $\lambda$ (ii) for $\alpha_{1}=\alpha_{2}, R=0.5, X_{1}$ and $X_{2}$ are independently and identically distributed and there is equal chance that $X_{1}$ is bigger than $X_{2}$.

\subsection{Estimation of Multicomponent Stress-Strength System Reliability for MBIII- Power Distribution}

Suppose a machine has at least " $s$ " components working out of " $m$ " component. The strengths of all the components of the system are $X_{1}, X_{2}, \ldots, X_{m}$ and stress $Y$ is applied to the system. Both strengths $X_{1}, X_{2}, \ldots, X_{m}$ and stress $Y$ are i.i.d. distributed. The cdf of $Y$ is $G$ and $F$ is the cdf of $X$. The reliability of a machine is the probability that the machine functions properly i.e.

$$
R_{s, m}=P(\text { strengths }>\text { stress })=P\left\{\text { at least " } s \text { " of }\left(X_{1}, X_{2}, \ldots, X_{m}\right) \text { exceed } Y\right\} .
$$

Let $X \sim$ MBIII-Power $\left(\alpha_{1}, \beta, \gamma, \kappa, \lambda\right), Y \sim$ MBIII-Power $\left(\alpha_{2}, \beta, \gamma, \kappa, \lambda\right)$ with unknown shape parameters $\alpha_{1}, \alpha_{2}$ and common shape $\beta, \gamma, \kappa$ and location parameter $\lambda$ where $X$, and $Y$ are independently distributed. The reliability in multicomponent stress- strength for MBIII-Power distribution 
is computed as:

$$
\begin{aligned}
R_{s, m}= & \sum_{l=s}^{m}\left(\begin{array}{c}
m \\
l
\end{array}\right) \int_{-\infty}^{\infty}[1-F(y)]^{l}[F(y)]^{m-l} d G(y) \quad \text { (Bhattacharyya and Johnson, 1974) } \\
= & \sum_{\ell=s}^{m}\left(\begin{array}{c}
m \\
l
\end{array}\right) \int_{0}^{\lambda}\left(1-\left[1+\gamma\left(\frac{x^{k}}{\lambda^{k}-x^{k}}\right)^{-\beta}\right]^{-\frac{\alpha_{1}}{\gamma}}\right)^{\ell}\left(\left[1+\gamma\left(\frac{x^{k}}{\lambda^{k}-x^{k}}\right)^{-\beta}\right]^{-\frac{\alpha_{1}}{\gamma}}\right)^{(m-\ell)} \\
& \times \frac{\alpha_{2} \beta k \lambda^{k} x^{-\beta k-1}}{\left(\lambda^{k}-x^{k}\right)^{-\beta+1}}\left[1+\gamma\left(\frac{x^{k}}{\lambda^{k}-x^{k}}\right)^{-\beta}\right]^{-\frac{\alpha_{2}}{\gamma}-1} d y
\end{aligned}
$$

Let $t=\left[1+\gamma\left(\frac{x^{k}}{\lambda^{k}-x^{k}}\right)^{-\beta}\right]^{-\frac{\alpha_{2}}{\gamma}}$, then we obtain $R_{s, m}=\sum_{\ell=s}^{m}\left(\begin{array}{c}m \\ l\end{array}\right) \int_{0}^{1}\left(1-t^{v}\right)^{\ell} t^{v(m-\ell)} d t$.

Let $z=t^{\nu}, t=z^{\frac{1}{\nu}}, d t=\frac{1}{\nu} z^{\frac{1}{\nu}-1} d z$, we reach at

$$
\begin{aligned}
R_{s, m} & =\sum_{\ell=s}^{m}\left(\begin{array}{c}
m \\
\ell
\end{array}\right) \int_{0}^{1}\left(1-t^{v}\right)^{\ell} t^{v(m-\ell)} d t \\
& =\sum_{\ell=s}^{m}\left(\begin{array}{c}
m \\
\ell
\end{array}\right) \int_{0}^{1}(1-z)^{\ell} z^{(m-\ell)} \frac{1}{\nu} z^{\frac{1}{\nu}-1} d z \\
& =\frac{1}{\nu} \sum_{\ell=s}^{m}\left(\begin{array}{c}
m \\
\ell
\end{array}\right) B\left(\ell+1, m-\ell+\frac{1}{\nu}\right) \\
& =\frac{1}{\nu} \sum_{\ell=s}^{m} \frac{m !}{(m-\ell) !}\left[\prod_{j=0}^{\ell}\left(m-j+\frac{1}{\nu}\right)\right]^{-1}
\end{aligned}
$$

where $v=\alpha_{2} / \alpha_{1}$. The probability $R_{s, m}$ in (3.3) is called multicomponent stress-strength model reliability.

\section{Characterization}

In this section, MBIII-Power distribution is characterized through: (i) conditional expectation; (ii) ratio of truncated moments; (iii) reverse hazard function and (iv) elasticity function.

\subsection{Characterization Based on Conditional Expectation}

Here MBIII-Power distribution is characterized via conditional expectation.

Proposition 4.1. Let $X: \Omega \rightarrow(0, \lambda)$ be a continuous random variable with cdf $F(x)(0<F(x)<$ 1 for $x \geq 0$ ), then for $\alpha>\gamma, X$ has cdf (2) if and only if

$$
E\left[\left(\frac{X^{\kappa}}{\lambda^{\kappa}-X^{\kappa}}\right)^{-\beta} \mid X<t\right]=\frac{1}{(\alpha-\gamma)}\left[1+\alpha\left(\frac{t^{\kappa}}{\lambda^{\kappa}-t^{\kappa}}\right)^{-\beta}\right] \text {, for } t>0 \text {. }
$$


Proof. If pdf of $X$ is (2.3), then

$$
\begin{aligned}
E\left[\left(\frac{X^{\kappa}}{\lambda^{\kappa}-X^{\kappa}}\right)^{\beta} \mid X<t\right] & =(F(t))^{-1} \int_{0}^{t}\left(\frac{x^{k}}{\lambda^{k}-x^{k}}\right)^{-\beta} f(x) d x \\
& =(F(t))^{-1} \int_{0}^{t}\left(\frac{x^{\kappa}}{\lambda^{\kappa}-x^{\kappa}}\right)^{-\beta} \frac{\alpha \beta k \lambda^{k} x^{-\beta k-1}}{\left(\lambda^{k}-x^{k}\right)^{-\beta+1}}\left[1+\gamma\left(\frac{x^{k}}{\lambda^{k}-x^{k}}\right)^{-\beta}\right]^{-\frac{\alpha}{\gamma}-1} d x
\end{aligned}
$$

Upon integration by parts and simplification, we obtain as

$$
E\left[\left(\frac{X^{\kappa}}{\lambda^{\kappa}-X^{\kappa}}\right)^{-\beta} \mid X<t\right]=\frac{1}{(\alpha-\gamma)}\left[1+\alpha\left(\frac{t^{\kappa}}{\lambda^{\kappa}-t^{\kappa}}\right)^{-\beta}\right] \quad \text { for } t>0 .
$$

Conversely, if proposition 6.1 .1 holds then

$$
\int_{0}^{t}\left(\frac{x^{\kappa}}{\lambda^{\kappa}-x^{\kappa}}\right)^{-\beta} f(x) d x=\frac{F(t)}{\gamma\left(\frac{\alpha}{\gamma}-1\right)}\left[1+\alpha\left(\frac{t^{\kappa}}{\lambda^{\kappa}-t^{\kappa}}\right)^{-\beta}\right] .
$$

Differentiating (4.1) with respect to $t$, we obtain

$$
\left(\frac{t^{\kappa}}{\lambda^{\kappa}-t^{\kappa}}\right)^{-\beta} f(t)=\frac{f(t)}{(\alpha-\gamma)}\left[1+\alpha\left(\frac{t^{\kappa}}{\lambda^{\kappa}-t^{\kappa}}\right)^{-\beta}\right]-\frac{F(t)}{\gamma\left(\frac{\alpha}{\gamma}-1\right)}\left[\frac{\alpha \beta \kappa \lambda^{\kappa} t^{-\beta \kappa-1}}{\left(\lambda^{\kappa}-t^{\kappa}\right)^{-\beta+1}}\right]
$$

After simplification and integration we arrive at $F(t)=\left[1+\gamma\left(\frac{t k}{\lambda^{k}-t^{k}}\right)^{\beta}\right]^{-\frac{\alpha}{\gamma}}, t \geq 0$.

\subsection{Characterization of MBIII-Power Distribution through Ratio of Trun- cated Moments}

Here MBIII-Power distribution is characterized using Theorem 1 (Glänzel; 1987) on the basis of a simple relationship between two truncated moments of $X$. Theorem 1 is given in Appendix A.

Proposition 4.2. Let $X: \Omega \rightarrow(0, \lambda)$ be a continuous random variable. Let

$$
h_{1}(x)=\frac{1}{\alpha}\left[1+\gamma\left(\frac{x^{\kappa}}{\lambda^{\kappa}-x^{\kappa}}\right)^{-\beta}\right]^{\frac{\alpha}{\gamma}+1}, x>0
$$

and $h_{2}(x)=2 \alpha^{-1}\left(\frac{x k}{\lambda^{k}-x^{k}}\right)^{-\beta}\left[1+\gamma\left(\frac{x k}{\lambda^{k}-x^{k}}\right)^{-\beta}\right]^{\frac{\alpha}{\gamma}+1}, x>0$. The random variable $X$ has $p d f(2.3)$ if and only if that the function $p(x)$ has the form $p(x)=\left(\frac{x^{\kappa}}{\lambda^{\kappa}-x^{\kappa}}\right)^{\beta}, x>0$. 
Proof. For random variable $X$ with pdf (2.3), then

$$
\begin{aligned}
& (1-F(x)) E\left(h_{1}(x) \mid X \geq x\right)=\left(\frac{x^{k}}{\lambda^{k}-x^{k}}\right)^{\beta} \\
& \text { and }(1-F(x)) E\left(h_{2}(x) \mid X \geq x\right)=\left(\frac{x^{k}}{\lambda^{k}-x^{k}}\right)^{2 \beta}, x>0 . \\
& \qquad \frac{E\left[h_{1}(x) \mid X \geq x\right]}{E\left[h_{2}(x) \mid X \geq x\right]}=p(x)=\left(\frac{x^{k}}{\lambda^{k}-x^{k}}\right)^{\beta}, x>0 \\
& \text { and } p^{\prime}(x)=\beta \kappa \lambda^{\kappa} x^{\kappa \beta-1}\left(\lambda^{\kappa}-x^{\kappa}\right)^{-\beta-1}, x>0 .
\end{aligned}
$$

The differential equation

$$
s^{\prime}(x)=\frac{p^{\prime}(x) h_{2}(x)}{p(x) h_{2}(x)-h_{1}(x)}=\frac{2 \beta \kappa \lambda^{\kappa} x^{\kappa-1}}{\left(\lambda^{\kappa}-x^{\kappa}\right)^{2}}\left(\frac{x^{\kappa}}{\lambda^{\kappa}-x^{\kappa}}\right)^{-1}
$$

has the solution

$$
s(x)=\ln \left(\frac{x^{\kappa}}{\lambda^{\kappa}-x^{\kappa}}\right)^{2 \beta}, x>0 .
$$

Therefore, in the light of Theorem 1, $X$ has the pdf (2.3)

Corollary 4.1. Let $X: \Omega \rightarrow(0, \lambda)$ be a continuous random variable and let

$$
h_{2}(x)=2 \alpha^{-1}\left(\frac{x^{\kappa}}{\lambda^{\kappa}-x^{\kappa}}\right)^{-\beta}\left[1+\gamma\left(\frac{x^{\kappa}}{\lambda^{\kappa}-x^{\kappa}}\right)^{-\beta}\right]^{\frac{\alpha}{\gamma}+1}, x>0 .
$$

The pdf of $X$ is (2.3) if and only if there exists functions $p(x)$ and $h_{1}(x)$ defined in Theorem 1, satisfying the differential equation

$$
\frac{p^{\prime}(x)}{p(x) h_{2}(x)-h_{1}(x)}=\alpha \beta \kappa \lambda^{\kappa} x^{\kappa \beta-1}\left(\lambda^{\kappa}-x^{\kappa}\right)^{-\beta-1}\left[1+\gamma\left(\frac{x^{\kappa}}{\lambda^{\kappa}-x^{\kappa}}\right)^{-\beta}\right]^{-\frac{\alpha}{\gamma}-1} .
$$

Remark 1. The solution of (4.2) is

$$
p(x)=\left(\frac{x^{\kappa}}{\lambda^{\kappa}-x^{\kappa}}\right)^{2 \beta}\left[\int\left(\frac{\alpha \beta \kappa \lambda^{\kappa} x^{-\beta \kappa-1}}{\left(\lambda^{\kappa}-x^{\kappa}\right)^{-\beta+1}}\left[1+\gamma\left(\frac{x^{\kappa}}{\lambda^{\kappa}-x^{\kappa}}\right)^{-\beta}\right]^{-\frac{\alpha}{\gamma}-1} h_{1}(x)\right) d x\right]+D
$$

where $\mathrm{D}$ is a constant. 


\subsection{Characterization of MBIII-Power Distribution Based on Reverse Hazard Rate Function}

Definition 4.1. Let $X: \Omega \rightarrow(0, \lambda)$ be a continuous random variable having absolutely continuous $F(x)$ and pdf $f(x)$, if and only if the reverse hazard function, $r_{F}(x)$, of a twice differentiable distribution function $F$, satisfying differential equation

$$
\frac{d}{d x}[\ln f(x)]=\frac{r_{F}^{\prime}(x)}{r_{F}(x)}+r_{F}(x) .
$$

Proposition 4.3. Let $X: \Omega \rightarrow(0, \lambda)$ be continuous random variable. The pdf of $X$ is (2.3) if and only if its reverse hazard function, $r_{F}$ satisfies the first order differential equation

$$
\mathrm{r}_{F}^{\prime}(\mathrm{x})\left[1+\gamma\left(\frac{x^{\kappa}}{\lambda^{\kappa}-x^{\kappa}}\right)^{-\beta}\right]-r_{F}(x) \frac{\beta \gamma \kappa \lambda^{\kappa} x^{-\beta \kappa-1}}{\left(\lambda^{\kappa}-x^{\kappa}\right)^{-\beta+1}}=\alpha \beta \kappa \lambda^{\kappa}\left[\frac{x^{\kappa}(\kappa+1)-(\beta \kappa+1) \lambda^{\kappa}}{x^{\beta \kappa+2}\left(\lambda^{\kappa}-x^{\kappa}\right)^{\beta}}\right]
$$

Proof. If $X$ has pdf (2.3), then (4.3) holds. Now if (4.3) holds, then

$$
\begin{aligned}
\frac{d}{d x}\left\{r_{F}(x)\left[1+\gamma\left(\frac{x^{\kappa}}{\lambda^{\kappa}-x^{\kappa}}\right)^{-\beta}\right]\right\} & =\frac{d}{d x}\left(\frac{\alpha \beta \kappa \lambda^{\kappa} x^{-\beta \kappa-1}}{\left(\lambda^{\kappa}-x^{\kappa}\right)^{-\beta+1}}\right) \\
\Rightarrow r_{F}(x) & =\frac{\alpha \beta \kappa \lambda^{\kappa} x^{-\beta \kappa-1}}{\left(\lambda^{\kappa}-x^{\kappa}\right)^{-\beta+1}}\left[1+\gamma\left(\frac{x^{\kappa}}{\lambda^{\kappa}-x^{\kappa}}\right)^{-\beta}\right]^{-1},
\end{aligned}
$$

which is reverse hazard function of MBIII-Power distribution.

\subsection{Characterization based on Elasticity Function}

In this sub-section, MBIII-Power distribution is characterized via elasticity.

Definition 4.2. Let $X: \Omega \rightarrow(0, \lambda)$ be a continuous random variable with cdf $F(x)$ and pdf $f(x)$, if and only if the elasticity function $e_{F}(x)$ of a twice differentiable distribution function $F$, satisfying the differential equation

$$
\frac{d}{d x}[\ln f(x)]=\frac{e^{\prime}(x)}{e(x)}+\frac{e(x)}{x}-\frac{1}{x} .
$$

Proposition 4.4. Let $X: \Omega \rightarrow(0, \lambda)$ be continuous random variable. The pdf of $X$ is $(2.3)$ provided that its elasticity function, $e_{F}(x)$ satisfies the first order differential equation

$$
\mathrm{e}_{F}^{\prime}(x)\left[1+\gamma\left(\frac{x^{\kappa}}{\lambda^{\kappa}-x^{\kappa}}\right)^{-\beta}\right]-e_{F}(x) \frac{\beta \gamma \kappa \lambda^{\kappa} x^{-\beta \kappa-1}}{\left(\lambda^{\kappa}-x^{\kappa}\right)^{-\beta+1}}=\alpha \beta \kappa^{2} \lambda^{\kappa}\left[\frac{x^{-\beta \kappa-1}\left(x^{\kappa}-\beta \lambda^{\kappa}\right)}{\left(\lambda^{\kappa}-x^{\kappa}\right)^{-\beta+2}}\right] .
$$

Proof. If $X$ has pdf (2.3), then (4.4) surely holds. Now if (4.4) holds, then

$$
\begin{aligned}
& \frac{d}{d x}\left\{e_{F}(x)\left[1+\gamma\left(\frac{x^{\kappa}}{\lambda^{\kappa}-x^{\kappa}}\right)^{-\beta}\right]\right\}=\frac{d}{d x}\left(\frac{\alpha \beta \kappa \lambda^{\kappa} x^{-\beta \kappa}}{\left(\lambda^{\kappa}-x^{\kappa}\right)^{-\beta+1}}\right) \\
\Rightarrow & e_{F}(x)=\frac{\alpha \beta \kappa \lambda^{\kappa} x^{-\beta \kappa}}{\left(\lambda^{\kappa}-x^{\kappa}\right)^{-\beta+1}}\left[1+\gamma\left(\frac{x^{\kappa}}{\lambda^{\kappa}-x^{\kappa}}\right)^{-\beta}\right]^{-1},
\end{aligned}
$$

which is elasticity function of MBIII-Power distribution. 


\section{Maximum Likelihood Estimation}

In this section, parameter estimates are derived using maximum likelihood method. The log likelihood function for MBIII-Power distribution with the parameter vector $\Phi=(\alpha, \beta, \gamma, \kappa, \lambda)^{\prime}$ is

$$
\begin{aligned}
\ln L\left(x_{i}, \Phi\right)= & n \ln \alpha+n \ln \beta+n \kappa \ln \lambda-(\kappa \beta+1) \sum_{i=1}^{n} \ln x_{i}-(\beta-1) \sum_{i=1}^{n} \ln \left(\lambda^{\kappa}-x_{i}^{\kappa}\right) \\
& -\left(\frac{\alpha}{\gamma}+1\right) \sum_{i=1}^{n} \ln \left[1+\gamma\left(\frac{x_{i}^{\kappa}}{\lambda^{\kappa}-x_{i}^{\kappa}}\right)^{-\beta}\right]
\end{aligned}
$$

where $\lambda$ is assumed to known because of its maximum likelihood is equal to maximum order statistics. In order to compute the maximum likelihood estimates (MLEs) of parameters of MBIII-Power distribution, the following nonlinear equations must be solved simultaneously:

$$
\begin{aligned}
\frac{\partial \ell(\Phi)}{\partial \alpha}= & \frac{n}{\alpha}-\frac{1}{\gamma} \sum_{i=1}^{n} \ln \left[1+\gamma\left(\frac{x_{i}^{k}}{\lambda^{k}-x_{i}^{k}}\right)^{-\beta}\right]=0 \\
\frac{\partial \ell(\Phi)}{\partial \beta}= & \frac{n}{\beta}-\sum_{i=1}^{n} \kappa \ln x_{i}-\sum_{i=1}^{n} \ln \left(\lambda^{\kappa}-x_{i}^{\kappa}\right)+(\alpha+\gamma) \sum_{i=1}^{n} \ln \left(\frac{x_{i}^{\kappa}}{\lambda^{\kappa}-x_{i}^{\kappa}}\right)\left[\left(\frac{x_{i}^{\kappa}}{\lambda^{\kappa}-x_{i}^{\kappa}}\right)^{\beta}+\gamma\right]^{-1}=0 \\
\frac{\partial \ell(\Phi)}{\partial \kappa}= & \frac{n}{\kappa}+n \ln (\lambda)+\beta \sum_{i=1}^{n} \ln x_{i}-(\beta-1) \sum_{i=1}^{n} \frac{\left(\lambda^{\kappa} \ln \lambda-x_{i}^{\kappa} \ln x_{i}\right)}{\left(\lambda^{\kappa-x_{i}^{\kappa}}\right)} \\
& -\beta(\alpha+\gamma) \sum_{i=1}^{n} \frac{\gamma\left(\left(\frac{x_{i}}{\lambda}\right)^{-\kappa}-1\right)^{\beta-1}\left(\frac{x_{i}}{\lambda}\right)^{-\kappa} \ln \left(\frac{\lambda}{x_{i}}\right)}{\left[1+\gamma\left(\frac{x_{i}^{\kappa}}{\lambda^{\kappa}-x_{i}^{\kappa}}\right)^{-\beta}\right]}=0 \\
\frac{\partial \ln L}{\partial \gamma}= & \frac{\alpha}{\gamma^{2}} \sum_{i=1}^{n} \ln \left[1+\gamma\left(\frac{x_{i}^{\kappa}}{\lambda^{\kappa}-x_{i}^{\kappa}}\right)^{-\beta}\right]-\left(\frac{\alpha}{\gamma}+1\right) \sum_{i=1}^{n}\left[\left(\frac{x_{i}^{\kappa}}{\lambda^{\kappa}-x_{i}^{\kappa}}\right)^{\beta}+\gamma\right]^{-1}=0 .
\end{aligned}
$$

\section{Simulation Study}

In this section, simulation study for the performance of the MLEs of the MBIII-Power parameters with respect to sample size $n$ is carried out. This performance is done based on the following simulation study: Step 1: Generate 10000 samples of size $\mathrm{n}$ from MBIII-Power distribution based on the Inverse CDF method. Step 2: Compute the MLEs for 10000 samples, say $(\hat{\alpha}, \hat{\beta}, \hat{\gamma}, \hat{\kappa}, \hat{\lambda})$ for $i=1,2, \ldots, 10000$ based on non-linear optimization algorithm with constraint matching to range of parameters. $(0.5,0.5,1.0,0.25,0.5)$ and $(0.8,0.75,1.5,0.5,0.25)$ are taken as the true parameter values $(\alpha, \beta, \gamma, \kappa, \lambda)$. Step 3: Compute the means, biases and mean squared errors of MLEs. For this purpose, we have selected different arbitrarily parameters and $n=10,50,100,150,200,250,500$ sample sizes. All codes are written in $\mathrm{R}$ and the results are summarized in Table 3. The results clearly show that when the sample size increases, the MSE of estimated parameters decrease and 
biases drop to zero. As shape parameter increases, MSE of estimated parameters increases. This shows the consistency of MLE estimators.

Table 3: Bias and MSEs of MBIII-Power distribution for $(0.5,0.5,1.0,0.25,0.5)$ and $(0.8,0.75,1.5,0.5,0.25)$

\begin{tabular}{ccccccc|ccccc}
\hline$n$ & Statistics & $\alpha=0.5$ & $\beta=0.5$ & $\gamma=1.0$ & $\kappa=0.25$ & $\lambda=0.5$ & $\alpha=0.8$ & $\beta=0.75$ & $\gamma=1.5$ & $\kappa=0.5$ & $\lambda=0.25$ \\
\hline 10 & Bias & .03 & 0.01 & 0.06 & 0.04 & -0.10 & 0.09 & -0.09 & 0.07 & 0.09 & -0.03 \\
& MSE & 0.02 & 0.03 & 0.43 & 0.03 & 0.05 & 1.00 & 0.03 & 13.51 & 0.04 & 0.00 \\
\hline 50 & Bias & 0.01 & 0.01 & 0.04 & 0.01 & -0.01 & 0.02 & -0.01 & 0.06 & 0.03 & -0.00 \\
& MSE & 0.00 & 0.00 & 0.00 & 0.00 & 0.00 & 0.03 & 0.01 & 0.09 & 0.01 & 0.00 \\
\hline 100 & Bias & 0.01 & 0.01 & 0.04 & 0.00 & -0.00 & 0.01 & 0.00 & 0.05 & 0.02 & -0.00 \\
& MSE & 0.00 & 0.00 & 0.00 & 0.00 & 0 & 0.01 & 0.00 & 0.03 & 0.01 & 0 \\
\hline 150 & Bias & 0.01 & 0.01 & 0.04 & 0.00 & 0.00 & 0.01 & 0.00 & 0.05 & 0.01 & -0.00 \\
& MSE & 0.00 & 0.00 & 0.00 & 0.00 & 0 & 0.01 & 0.00 & 0.01 & 0.00 & 0 \\
\hline 200 & Bias & 0.01 & 0.01 & 0.03 & 0.00 & 0.00 & 0.01 & 0.01 & 0.05 & 0.01 & 0.00 \\
& MSE & 0.00 & 0.00 & 0.00 & 0.00 & 0 & 0.00 & 0.00 & 0.01 & 0.00 & 0 \\
\hline 250 & Bias & 0.01 & 0.01 & 0.03 & 0.00 & 0.00 & 0.01 & 0.01 & 0.05 & 0.01 & 0.00 \\
& MSE & 0.001 & 0.00 & 0.00 & 0.00 & 0 & 0.00 & 0.00 & 0.01 & 0.00 & 0 \\
\hline \multirow{2}{*}{500} & Bias & 0.00 & 0.00 & 0.03 & 0.00 & 0.00 & 0.01 & 0.00 & 0.04 & 0.00 & 0.00 \\
& MSE & 0.00 & 0.00 & 0.00 & 0.00 & 0 & 0.00 & 0.00 & 0.01 & 0.00 & 0 \\
\hline
\end{tabular}

\section{Applications}

In this section, use of MBIII-Power distribution is demonstrated by applying it to two data sets: serum-reversal time (in days) of children born from HIV-infected mothers (Lee, 1992) and failure times of devices(Aarset, 1987) data. Goodness of fit of the probability distribution through different methods is studied. The MBIII-Power distribution is compared with sub-models such as BIII-Power, Inverse Lomax-Power (IL-Power), LL-Power, generalized inverse Weibull-Power (GIW-Power) and competing models such as modified Burr XII (MBXII), Modified Burr III (MBIII), Weibull and inverse Weibull distributions. The maximum likelihood estimates (MLEs) of unknown parameters and values of goodness of fit measures such as "Cramer-von Mises (W), Anderson Darling (A), Kolmogorov- Smirnov (K-S) statistics with p-values, Akaike information criterion (AIC), consistent Akaike information criterion (CAIC), Bayesian information criterion (BIC), Hannan-Quinn information criterion (HQIC) and likelihood ratio statistics" are computed for using R-package for serum-reversal time (in days) of children born from HIV-infected mothers and failure times of devices data. The better fit corresponds to smaller W, A, K-S, AIC, CAIC, BIC, HQIC and value. The MLEs, their standard errors (in parentheses) and goodness-of-fit statistics such as W, A, K-S 
(p-value) are given in Tables 4 and 6. Table 5 displays goodness-of-fit values.

Table 4: MLEs, their standard errors (in parentheses) and Goodness-of-fit statistics for SerumReversal Time of Children Data

\begin{tabular}{|c|c|c|c|c|c|c|c|c|}
\hline Model & $\alpha$ & $\beta$ & $\gamma$ & $\kappa$ & $\lambda$ & $\mathrm{W}$ & A & $\begin{array}{c}\text { K-S p-value } \\
\text { p-value }\end{array}$ \\
\hline MBIII-Power & $\begin{array}{c}42.70 \\
(137.84)\end{array}$ & $\begin{array}{c}2.55 \\
(0.33)\end{array}$ & $\begin{array}{c}70.02 \\
(209.60)\end{array}$ & $\begin{array}{c}0.26 \\
(0.30)\end{array}$ & 1021 & 0.16 & 1.01 & $\begin{array}{c}0.06 \\
(0.60)\end{array}$ \\
\hline BIII-Power & $\begin{array}{c}0.34 \\
(0.08)\end{array}$ & $\begin{array}{c}2.17 \\
(0.27)\end{array}$ & 1 & $\begin{array}{c}1.32 \\
(0.18)\end{array}$ & 1021 & 0.28 & 1.61 & $\begin{array}{c}0.10 \\
(0.13)\end{array}$ \\
\hline IL-Power & $\begin{array}{c}0.22 \\
(3.20)\end{array}$ & 1 & 1 & $\begin{array}{c}3.70 \\
(53.34)\end{array}$ & 1021 & 0.67 & 3.68 & $\begin{array}{c}0.20 \\
(<.0001)\end{array}$ \\
\hline LL-Power & 1 & $\begin{array}{l}1.55 \\
(0.11)\end{array}$ & 1 & $\begin{array}{c}0.72 \\
(0.04)\end{array}$ & 1021 & 0.54 & 2.98 & $\begin{array}{c}0.11 \\
(0.04812)\end{array}$ \\
\hline GIW-Power & $\begin{array}{c}178.19 \\
(145.93)\end{array}$ & $\begin{array}{l}1.23 \\
(0.07)\end{array}$ & 1 & $\begin{array}{c}0.01 \\
(0.01)\end{array}$ & 1021 & 0.82 & 4.53 & $\begin{array}{c}0.14 \\
(0.01)\end{array}$ \\
\hline MBXII & $\begin{array}{l}82380.00 \\
(2914.94)\end{array}$ & $\begin{array}{c}0.16 \\
(0.01)\end{array}$ & $\begin{array}{l}76069.00 \\
(1507.75)\end{array}$ & - & - & 2.58 & 14.02 & $\begin{array}{c}1 \\
(<.0001)\end{array}$ \\
\hline MBIII & $\begin{array}{l}82380.39 \\
(261.14)\end{array}$ & $\begin{array}{c}1.91 \\
(0.02)\end{array}$ & $\begin{array}{l}76069.29 \\
(1857.72)\end{array}$ & - & - & 1.76 & 9.75 & $\begin{array}{c}0.14 \\
(0.01)\end{array}$ \\
\hline Weibull & $\begin{array}{c}0.002 \\
(0.0002)\end{array}$ & $\begin{array}{c}1.02 \\
(0.02)\end{array}$ & - & - & - & 1.20 & 6.74 & $\begin{array}{c}0.27 \\
(<.0001)\end{array}$ \\
\hline Inverse Weibull & $\begin{array}{l}16.73 \\
(2.36)\end{array}$ & $\begin{array}{c}0.56 \\
(0.03)\end{array}$ & - & - & - & 4.01 & 20.98 & $\begin{array}{c}0.29 \\
(<.0001)\end{array}$ \\
\hline
\end{tabular}

The MBIII-Power distribution is best fitted model than the other models because the values of all criteria of goodness of fit are smaller for MBIII-Power distribution. We can also perceive that the MBIII-Power distribution is best fitted model than other models because MBIII-Power distribution offers the closer fit to empirical data (Figure 3). The MBIII-Power distribution is best fitted model than the other models because the values of all criteria of goodness of fit are smaller for MBIIIPower distribution. We can also perceive that the MBIII-Power distribution is best fitted model than other models because MBIII-Power distribution offers the closer fit to empirical data (Figure 3). 
Table 5: Goodness-of-fit statistics for the fitted mode for the two data sets considered for the applications

\begin{tabular}{llccccc}
\hline Data & Model & AIC & CAIC & BIC & HQIC & $-\ell$ \\
\hline \multirow{2}{*}{ Children } & MBIII-Power & 1922.35 & 1922.65 & 1934.18 & 1927.16 & 957.18 \\
& BIII-Power & 1925.94 & 1926.12 & 1934.81 & 1929.55 & 959.97 \\
& IL-Power & 1965.69 & 1965.78 & 1971.61 & 1968.10 & 980.85 \\
& LL-Power & 1936.57 & 1936.66 & 1942.48 & 1938.97 & 966.28 \\
& GIW-Power & 1956.99 & 1957.16 & 1965.86 & 1960.59 & 975.49 \\
& MBXII & 5906.84 & 5907.01 & 5915.73 & 5910.45 & 2950.42 \\
& MBIII & 2046.30 & 2046.47 & 2055.19 & 2049.91 & 1020.15 \\
& Weibull & 2018.20 & 2018.28 & 2024.12 & 2020.61 & 1007.10 \\
& Inverse Weibull & 2202.32 & 2202.40 & 2208.24 & 2204.73 & 1099.16 \\
\hline \multirow{2}{*}{ Failure time } & MBIII-Power & 399.54 & 400.47 & 407.02 & 402.37 & 195.77 \\
& BIII-Power & 405.55 & 406.10 & 411.17 & 407.67 & 199.78 \\
& IL-Power & 424.58 & 424.85 & 428.33 & 425.10 & 210.29 \\
& LL-Power & 403.70 & 403.96 & 407.44 & 405.11 & 199.85 \\
& GIW-Power & 405.32 & 405.87 & 410.94 & 407.44 & 199.66 \\
MBXII & 499.42 & 499.93 & 505.22 & 501.64 & 246.71 \\
& MBIII & 487.86 & 488.37 & 493.66 & 490.08 & 240.93 \\
Weibull & 497.42 & 497.67 & 501.29 & 498.90 & 246.71 \\
Inverse Weibull & 547.26 & 547.51 & 551.13 & 548.74 & 271.63 \\
\hline & & & & & \\
\hline
\end{tabular}
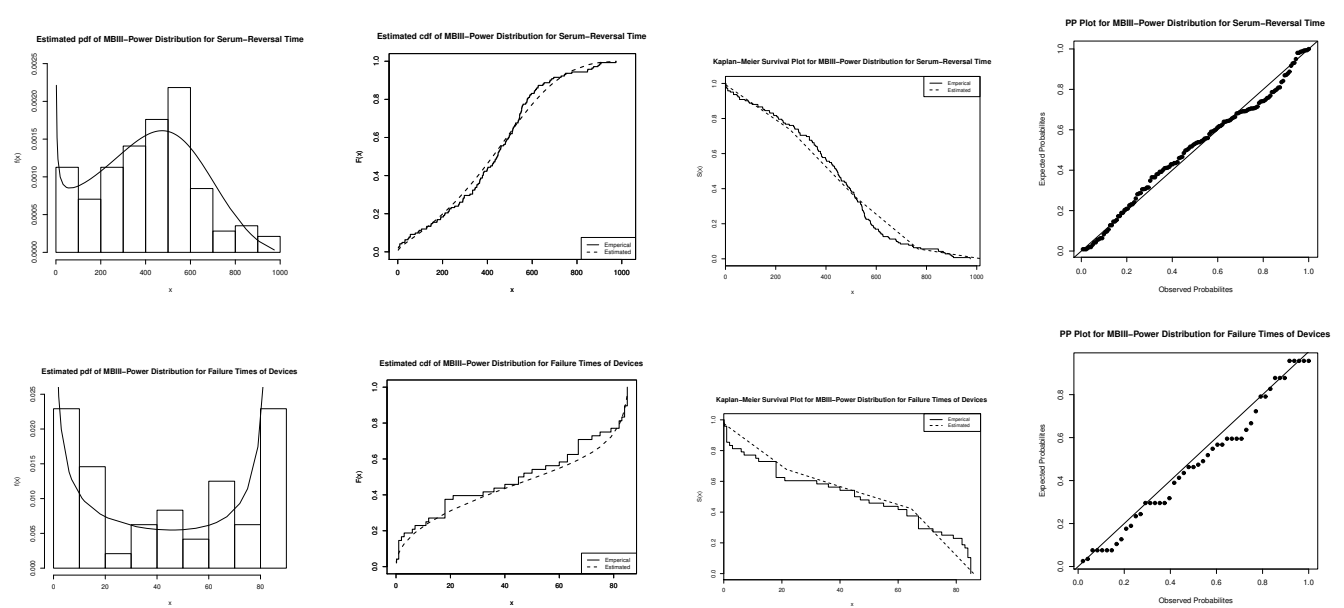

Figure 3: Fitted pdf, cdf, survival and pp plots of MBIII-Power distribution for Serum-Reversal Time of Children Data (first row) and Failure time data (second row) 
Table 6: MLEs, their standard errors (in parentheses) and Goodness-of-fit statistics for Failure Times

\begin{tabular}{|c|c|c|c|c|c|c|c|c|}
\hline Model & $\alpha$ & $\beta$ & $\gamma$ & $\kappa$ & $\lambda$ & W & A & $\begin{array}{c}\mathrm{K}-\mathrm{S} \\
\mathrm{p} \text {-value }\end{array}$ \\
\hline \multirow[t]{2}{*}{ MBIII-Power } & 7411.98 & 3.55 & 186787.5 & 3.35 & 86.0 & 0.05 & 0.41 & 0.11 \\
\hline & (2327.91) & $(0.38)$ & $(3710.51)$ & (1.19) & & & & $(0.57)$ \\
\hline \multirow[t]{2}{*}{ BIII-Power } & 1.19 & 0.59 & 1 & 0.78 & 86.0 & 0.05 & 0.49 & 0.08 \\
\hline & $(0.56)$ & (0.09) & & $(0.50)$ & & & & $(0.93)$ \\
\hline \multirow[t]{2}{*}{ IL-Power } & 1.10 & 1 & 1 & 0.64 & 86.0 & 0.07 & 0.58 & 0.22 \\
\hline & $(69.21573)$ & & & (40.21927) & & & & $(0.02)$ \\
\hline \multirow[t]{2}{*}{ LL-Power } & 1 & 0.57 & 1 & 0.97 & 86.0 & 0.06 & 0.51 & 0.08 \\
\hline & & $(0.08)$ & & $(0.20)$ & & & & $(0.91)$ \\
\hline \multirow[t]{2}{*}{ GIW-Power } & 1.34 & 0.51 & 1 & 0.35 & 86.0 & 0.059 & 0.51 & 0.08 \\
\hline & $(0.68)$ & $(0.09)$ & & $(0.20)$ & & & & $(0.93)$ \\
\hline \multirow[t]{2}{*}{ MBXII } & 171.99 & 5.28 & 4243.40 & - & - & 1.35 & 7.10 & 0.36 \\
\hline & (247.53) & $(0.96)$ & $(6608.93)$ & & & & & $(<.0001)$ \\
\hline \multirow[t]{2}{*}{ MBIII } & 1461412 & 3.48 & 7138308 & - & - & 0.41 & 2.56 & 0.17 \\
\hline & $(6.60)$ & $(0.09)$ & (13699.08) & & & & & $(0.09)$ \\
\hline \multirow[t]{2}{*}{ Weibull } & 0.03 & 0.96 & - & - & - & 0.53 & 3.17 & 0.20 \\
\hline & $(0.01)$ & $(0.12)$ & & & & & & $(0.04)$ \\
\hline \multirow[t]{2}{*}{ Inverse Webill } & 2.68 & 0.46 & - & - & - & 1.09 & 5.80 & 0.29 \\
\hline & $(0.39)$ & $(0.04)$ & & & & & & (0.0004) \\
\hline
\end{tabular}

\section{Concluding Remarks}

We have developed the MBIII-Power distribution on the basis of the generalized Pearson differential equation. We have studied properties such as sub-models; descriptive measures based on the quantiles, moments, inequality measures, residual and reverse residual life function, stress-strength reliability and multicomponent stress-strength reliability model. The MBIII-Power distribution is characterized via different techniques. The MLEs for MBIII-Power distribution have been computed. The simulation study for performance of the maximum likelihood estimates (MLEs) of parameters for the MBIII-Power distribution has been carried out. Applications of the MBIII-Power model to real data sets (serum-reversal time (in days) of children born from HIV-infected mothers and failure times of devices data) are presented to show the significance and flexibility of MBIIIPower distribution. Goodness of fit shows that the MBIII-Power distribution is a better fit. We have proved that MBIII-Power distribution is empirically better for lifetime applications. 


\section{Acknowledgement}

Author is thankful to editor and reviewers for valuable suggestions for improving the article.

\section{References}

Aarset, M. V. (1987), "How to identify bathtub hazard rate", IEEE Transactions on Reliability, 36, 106-108.

Ali, A., Husnain, S. A., and Ahmad, M. (2015), "Modified Burr III distribution, Properties and Applications”, Pakistan Journal of Statistics, 31(6), 697-708.

Benjamin, S. M., Humberto, V. H., and Arnold, B.C. (2013), "Use of the Dagum Distribution for Modeling Tropospheric Ozone levels", J. Env. Stat., 5(6), 1-11.

Bertand, J., Bertand, P., and Ovarlez, J. P. (1994), “The mellin Transform”, ONERA, TP, 199498.

Bhattacharyya, G. K. and Johnson, R. A. (1974), "Estimation of reliability in a multicomponent stress-strength model", Journal of the American Statistical Association, 69, 966-970.

Burr, I. W. (1942), “Cumulative frequency functions”, The Annals of Mathematical Statistics, 13, 215-232.

Cordeiro, G. M., Gomes, A. E., da-Silva, C. Q., and Otega, E. M. (2017), “A useful extension of the Burr III distribution", Journal of Statistical Distributions and Applications, 4(1), 24.

Dagum, C. A. (1977), "New model of personal income distribution: specification and estimation”, Eco. Apl., 30, 413-437.

Dunning, K. A. and Hanson, J. N. (1977), “Generalized Pearson distributions and nonlinear programing”, Journal of Statistical Computation and Simulation, 6(2), 115-128.

Glänzel, W. A. (1990), "Some consequences of a characterization theorem based on truncated moments", Statistics, 21, 613-618.

Gomes, A. E., da-Silva, C. Q., and Cordeiro, G. M. (2015), "Two extended Burr models: Theory and practice", Communications in Statistics-Theory and Methods, 44(8), 1706-1734.

Gove, J. H., Ducey, M. J., Leak, W. B., and Zhang, L. (2008), "Rotated sigmoid structures in managed uneven-aged northern hard work stands: a look at the Burr Type III distribution", Foresty, 81(2),161-176.

Kleiber, C. and Kotz, S. (2003), "Statistical size distribution in economics and actuarial sciences", John Wiley and Sons, New York.

Lee, E. T. (1992), “Statistical Methods for Survival Data Analysis”, John Wiley, New York. 
Mielke, P. W. (1973), "Another family of distributions for describing and analyzing precipitation data", J. Appl. Meterol., 12, 275-280.

Moisheer, A. S. A. (2016), "A mixture of two Burr Type III distributions: Identifiability and estimation under type II censoring”, Mathematical Problems in Engineering, 7035279.

Olobatuyi, K. I., Asiribo, O. E., and Dawodu, G. A. (2017), “A New Class of Generalized Burr III Distribution for Lifetime Data", arXiv preprint arXiv:1701.00403.

Shao, Q., Chen, Y. D., and Zhang, L. (2008), "An extension of three-parameter Burr III distribution for low-flow frequency analysis", Comput. Stat. Data Anal., 52, 1304-1314.

Received: April 24, 2018

Accepted: November 16, 2018 